\title{
RAPID RECOGNITION OF TOMATO’S DiSEASE STAGES BASED ON THE KERNEL MutuAl SUBSPACE METHOD
}

\author{
Yan Zhang ${ }^{1}$, Huarui $\mathrm{Wu}^{1}$, Cheng Chen ${ }^{1, *}$, Xiaomin Wang ${ }^{1}$ \\ 1 National Engineering Research Center for Information Technology in Agriculture, \\ Beijing Research Center for Information Technology in Agriculture, and Key Laboratory of Agri-informatics, \\ Ministry of Agriculture and Rural Affairs, Haidian District, Beijing, China. \\ * Correspondence: nercitabdi@163.com.
}

\section{HighLIGHTS}

- A low time complexity and high accuracy recognition method of tomato's disease stages was proposed.

- Small number of disease image samples can support a stable and high accuracy method with less computation time and less memory cost.

- Provided a solution to establish a real-time and low energy consumption disease recognize system.

ABSTRACT. Disease stages recognition of tomato is important for the timely diagnosis and prevention of tomato diseases. In this article, the color and texture features were extracted, and the kernel mutual subspace method (KMSM) was introduced to establish a rapid Tomato's disease stage recognition method. Firstly, the color and textural features were extracted from tomato leaf and mapped to a high-dimensional space using a Gaussian kernel function. Secondly, a nonlinear disease feature subspace was established by applying principal component analysis (PCA) based on the mapped high-dimensional space. Finally, disease stages are recognized by calculating the canonical angles between the testing subspace and reference sub-spaces. To validate recognition rate of the proposed method, we conducted 10-fold cross-validation for experiments using the tomato disease sets contained in PlantVillage and artificial intelligence (AI) Challenger 2018 datasets. Also compared with the support vector machine (SVM) and VGG 16 methods, the results show that, accuracy of those three evaluated methods on the PlantVillage datasets are 99.34\%, 89.2\%, and 97\%, respectively; and accuracy on the AI Challenger 2018 datasets are $98.66 \%, 71.14 \%$, and $73.93 \%$, respectively. Moreover, average training and recognition time of the proposed method is 0.1496 and $0.008 \mathrm{~s}$, respectively, which is faster than SVM and VGG16 methods. In conclusion, the proposed method can be carried out in real-time recognition intelligent equipment which requires less memory space, less computation time, and with higher recognition rate, and low energy consumption.

Keywords. Color and textural feature, High dimensional feature space, Kernel mutual subspace method, Tomato's disease stages recognition,.

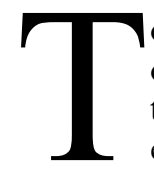
omatoes are a widely planted vegetable throughout the world, with the decreased quality and yield, the economic profit of is impacted by untimely diseases (Barbedo and Arnal, 2016; Kumar and Vani, 2019; Liu and Wang, 2020; Luna-Benoso et al., 2021). There are all kinds of tomato leaf diseases, such as tomato yellow leaf curl disease and powdery mildew, it's difficult to make a distinction (Juan et al., 2010; Mabvakure et al., 2016; Canizares et al., 2015). In a traditional agricultural production process, different stages of tomatoes disease are judged by experience or experts, which often leads to misdiagnosis. Besides, extensive experience is required for tomato disease stage judgement, and supports precision pesticide spray ap-

Submitted for review on 28 January 2021 as manuscript number ITSC 14507; approved for publication as a Research Article and as part of the Computer Modeling and Statistics for Agriculture Collection by the Information Technology, Sensors, \& Control Systems Community of ASABE on 31 July 2021. plication with high demand skills. Due to fast spread of diseases, the best control period for tomato diseases is short (Fuentes et al., 2018; Karthik et al., 2020). By means of computer-vision technology, tomato leaf spot features are extracted and identified automatically (Rangarajan et al., 2018; $\mathrm{Tm}$ et al., 2018), subjectivity of the diagnosis and dependence on experts are eliminated, and the diseases are identified in a timely and accurate approach. Precise pesticide spraying can control diseases and pests, but they also greatly reduce the dosage of pesticides and reduce environmental pollution. Plant diseases usually come with different symptoms, such as color change, shapes and texture change, which can be extracted and utilized by computer version (Deng et al., 2016; Everton et al., 2017 Hassanien et al., 2017; Pantazi et al., 2019; Kasinathan and Uyyala, 2021). In recent years, the research on the application of machine learning and deep learning methods in disease identification has made great progress and achievement, such as the support vector machine (Deng et al., 2016; Hassanien et al., 2017; Kasinathan and Uyyala, 2021), K-NN (Deng et al., 
Table 1. Summary of proposed models and accuracy in disease recognition.

\begin{tabular}{|c|c|c|c|c|c|}
\hline Author & Classifiers & $\begin{array}{l}\text { Features Extraction/ } \\
\text { Training Mechanism }\end{array}$ & $\begin{array}{c}\text { Accuracy of } \\
\text { the Model }\end{array}$ & Merit & Demerits \\
\hline \multicolumn{6}{|l|}{ Conventional Methods } \\
\hline $\begin{array}{l}\text { Luna-Benoso } \\
\text { et al. (2021) }\end{array}$ & $\begin{array}{c}\text { Three classifiers } \\
\text { (MLP, K-NN, SVM) } \\
\text { are used to decide the } \\
\text { output by means } \\
\text { of a decision rule. }\end{array}$ & $\begin{array}{l}\text { GLCM Gray Level } \\
\text { Co-occurrence Matrix } \\
\text { (GLCM) and } \\
\text { Color moments. }\end{array}$ & $\begin{array}{c}86.45 \% \text { (Healthy } \\
\text { leaves and } \\
\text { diseased leaves), } \\
97.39 \% \text { (type } \\
\text { of diseases). }\end{array}$ & $\begin{array}{l}\text { High accuracy } \\
\text { with the type } \\
\text { of disease. }\end{array}$ & $\begin{array}{c}\text { Low accuracy } \\
\text { on healthy } \\
\text { and diseased. }\end{array}$ \\
\hline $\begin{array}{l}\text { Pantazi et al. } \\
(2019)\end{array}$ & One Class Classifier. & $\begin{array}{c}\text { Local Binary } \\
\text { Patterns (LBPs). }\end{array}$ & $95 \%$ & $\begin{array}{c}\text { High } \\
\text { generalization } \\
\text { behavior. }\end{array}$ & $\begin{array}{l}\text { Has not been } \\
\text { extended to crop } \\
\text { status detection. }\end{array}$ \\
\hline $\begin{array}{l}\text { Deng et al. } \\
(2016)\end{array}$ & C-SVC. & $\begin{array}{c}\text { Texture and } \\
\text { histogram of } \\
\text { the color space } \\
\text { (gray and HSI) }\end{array}$ & $91.93 \%$ & $\begin{array}{c}\text { Low cost and } \\
\text { low computational } \\
\text { complexity. }\end{array}$ & $\begin{array}{l}\text { Lower accuracy } \\
\text { of classification. }\end{array}$ \\
\hline $\begin{array}{l}\text { Hassanien et al. } \\
(2017)\end{array}$ & $\mathrm{KNN}$ & $\begin{array}{l}\text { Moth-Flame } \\
\text { Optimization } \\
\text { and rough set } \\
\text { (MFORSFS) }\end{array}$ & $91.5 \%$ & $\begin{array}{c}\text { Robustness } \\
\text { and faster } \\
\text { convergence. }\end{array}$ & Low accuracy. \\
\hline $\begin{array}{l}\text { Kasinathan } \\
\text { and Uyyala } \\
(2021)\end{array}$ & $\begin{array}{c}\text { Base classifiers and } \\
\text { ensemble classifiers } \\
\text { were applied to decide } \\
\text { the output by majority } \\
\text { voting. } \\
\text { Base classifiers: } \\
\text { Naive Bayes (NB), } \\
\text { SVM, KNN and MLP; } \\
\text { Ensemble classifiers: } \\
\text { Random forest (RF), } \\
\text { Bagging and XGBoost. }\end{array}$ & $\begin{array}{c}\text { The combination } \\
\text { of texture, } \\
\text { color, shape, } \\
\text { HOG and GIST. }\end{array}$ & $\begin{array}{c}\text { High } \\
\text { classification } \\
\text { and } \\
\text { identification } \\
\text { of insects. }\end{array}$ & $\begin{array}{l}\text { Accuracy is } \\
\text { improved by } \\
\text { majority voting } \\
\text { with ensemble } \\
\text { classifiers. }\end{array}$ & NIL \\
\hline \multicolumn{6}{|l|}{$\overline{\text { CNN-based Methods }}$} \\
\hline $\begin{array}{l}\text { Liu and Wang } \\
(2020)\end{array}$ & $\begin{array}{c}\text { MobileNet } \\
\text { V2-YOLOV3. }\end{array}$ & $\begin{array}{l}\text { Mixed training } \\
\text { and transfer } \\
\text { learning. }\end{array}$ & $\begin{array}{c}93.24 \%(\mathrm{~F} 1), \\
91.32 \%(\mathrm{AP}), \\
86.98 \%(\mathrm{IOU}) .\end{array}$ & $\begin{array}{l}\text { Achieved a good } \\
\text { balance between } \\
\text { accuracy and } \\
\text { real-time detection. }\end{array}$ & $\begin{array}{l}\text { Accuracy is } \\
\text { reduced. }\end{array}$ \\
\hline $\begin{array}{l}\text { Kumar and Vani } \\
(2019)\end{array}$ & $\begin{array}{l}\text { 1. LeNet; } \\
\text { 2. VGGNet; } \\
\text { 3. ResNet50; } \\
\text { 4. Xception. }\end{array}$ & $\begin{array}{l}\text { Training } \\
\text { from } \\
\text { scratch }\end{array}$ & $\begin{array}{c}99.25 \% \text { (fine } \\
\text { tuned VGGNet). }\end{array}$ & High accuracy & $\begin{array}{l}\text { Consumes much } \\
\text { time and requires } \\
\text { high end hardware } \\
\text { configuration. }\end{array}$ \\
\hline $\begin{array}{l}\text { Fuentes et al. } \\
(2018)\end{array}$ & $\begin{array}{l}\text { 1. FRCNN; } \\
\text { 2. Refinement } \\
\text { Filter Bank. }\end{array}$ & $\begin{array}{l}\text { Training } \\
\text { from } \\
\text { scratch. }\end{array}$ & $\begin{array}{c}96.25 \% \\
\text { (Mean AP). }\end{array}$ & High accuracy. & $\begin{array}{c}\text { The processing } \\
\text { stage takes } \\
\text { much time. }\end{array}$ \\
\hline $\begin{array}{l}\text { Rangarajan et al. } \\
(2018)\end{array}$ & $\begin{array}{l}\text { 1. AlexNet; } \\
\text { 2. VGG16. }\end{array}$ & $\begin{array}{l}\text { Pre-trained; } \\
\text { Fine tuning. }\end{array}$ & $\begin{array}{l}97.29 \% \text { (VGG16), } \\
97.49 \% \text { (AlexNet). }\end{array}$ & $\begin{array}{l}\text { High accuracy } \\
\text { on AlexNet. }\end{array}$ & $\begin{array}{c}\text { The number of } \\
\text { images significantly } \\
\text { affected the } \\
\text { performance of } \\
\text { the models. }\end{array}$ \\
\hline
\end{tabular}

2016), ensemble learning (Fuentes et al., 2018; Rangarajan et al., 2018), and many other methods. Table 1 summarizes previous work and accuracy in disease recognition.

Studies achieve great recognition rates for plant diseases with the use of one or more classifiers. Most of the work shown in the table take advantage of a one-classifier model such as CNN, SVM, K-NN, and MLP among others; other work propose to carry out a model with multi-classifiers and decide the output through a decision rule or majority voting (Luna-Benoso et al., 2021; Kasinathan and Uyyala, 2021), which achieve better results than one-classifier model with the sacrifice of time. In this article, we proposed a one-classifier method which can achieve better accuracy than multiclassifiers model with less computation time.

Although the CNN-based method has achieved good results in plant disease detection as a robust method, there are still many constraints, including: (1) The system is complex and has a large amount of computation, which requires high requirements on the operating platform and operating environment, and usually requires a GPU computing core; (2) A large number of high-resolution image are needed, and the time complexity of training and recognition is high, which cannot meet the standard of the real-time system at present.

On the other hand, compared with deep learning methods, the advantages of machine learning method are mainly reflected in the following aspects: (1) The requirement of running platform and running environment is not high, and the system could run in the conventional computer and terminal equipment; (2) Small sample data set could achieve high recognition accuracy, and the training time and recognition time are shorter, which could meet the standard of real-time system.

As the training and recognition process of KMSM model are not dependent on GPU, the low-configuration system could also practice real-time operation. At the same time, the model generates the principal component features of the recognized objects based on the image set, which is conducive 
to the recognition of local shape differences. Therefore, in this article, we employed the KMSM to classify the different stages of tomato diseases (early stage and advanced stage) to realize the accurate and rapid recognition. So far, there are many researches for disease recognition, but few researches conducted for different stages disease detection, the early stage tomato disease reveals weak features and is challenging to identify. Accurate identification of diseases at early stage is critical for timely prevention, and further reduce productive losses.

The methodology is divided into three stages: Firstly, the low-dimensional color features of disease leaves are extracted, which including the color moment $(\mathrm{CM})$, color coherence vector $(\mathrm{CCV})$ and histogram of oriented gradient (HOG). Secondly, the kernel mutual subspace method (KMSM) is utilized to map the extracted features to a highdimensional feature subspace and established an nonlinear tomato disease feature subspace. Finally, by calculating the minimum regular angle between the training and testing nonlinear feature subspace, disease can be recognized and classified into different stages (early stage and advanced stage). The model takes full advantage of the robustness of KMSM to the image shooting angle and illumination conditions, combined with the color and texture characteristics of tomato leaves, it can realize rapid and efficient tomato leaf disease identification, and small size disease image samples can support stable and high accuracy system, with less computational cost and storage requirement.

\section{MATERIALS AND MeTHODS \\ DATASETS}

Plant Village (Hughes and Salathe, 2015) is a disease type datasets that includes 10 types: tomato bacterial spot (TBS), tomato early blight (TEB), healthy tomato (TH), tomato late blight (TLB), tomato leaf mold (TLM), tomato septoria leaf spot blight (TSL), tomato spider mites two spots spider mite (TSM), tomato target spot bacteria (TTS), tomato mosaic virus (TMV) and tomato yellow leaf curl virus (TYV). All images in the datasets are taken under natural light conditions with simple backgrounds. Each picture contains only one tomato leaf, and the leaf is located at the center of the picture.

AI Challenger 2018 (Singkek sci-tech, 2020) (https://challenger.ai/competition/pdr2018) is a disease stage datasets that includes nine groups of diseases with general (early) and serious (advanced) stages, and one tomato mosaic virus and one healthy class. The image collection method is the same as that in PlantVillage. After preprocessing the datasets of tomato diseases with 14 categories was extracted from AI Challenger 2018, includes:

- healthy tomato (TH),

- tomato early blight fungus general (TEBg), tomato early blight fungus serious (TEBs),

- tomato late blight water mold general (TLBg), tomato late blight water mold serious (TLBs),

- tomato leaf mold fungus general (TLMg), tomato leaf mold fungus serious (TLMs),

- tomato Septoria leaf spot fungus general (TSLg), tomato Septoria leaf spot fungus serious (TSLs),
- tomato spider mite damage general (TSMg), tomato spider mite damage serious (TSMs),

- tomato yellow leaf curl virus general (TYVg), tomato yellow leaf curl virus serious (TYVs),

- tomato mosaic virus (TMV).

\section{COlOR AND TeXture Feature EXTRACTiON}

As leaf diseases often show changes in the colors, shapes and textures, which are wildly utilized for plant disease classify. The color features extracted in this article include the RGB color moments (CM) (Stricker and Orengo, 1995) and the color coherence vector (CCV) (Pass et al., 1997). The textural features extracted in this article are using the histogram of oriented gradients (HOG) (Dalal and Triggs, 2005).

\section{Color Feature Extraction}

At different disease stage, the disease leaf and spots on it may express different colors; usually leaf spot with an advanced stage disease express darker color. Figure 1 shows TEBg - tomato early blight fungus (general-early stage and serious-advanced stage) images in AI Challenger 2018. At the early stage of TEBg, small black spots appeared on the leaves and gradually expand to concentric ring spots with yellow or light green halos around; at the late stage of TEBs, irregular round or oval spots of dark brown accompanied by gray black mold. It can be found that, the color feature is acute to distinguish the disease types and stages, which can utilize color feature for early detection of tomato diseases.

The color moment (CM) was proposed by Stricker and Orengo (1995), was verified as an effective color feature. This method uses the concept of the moment in linear algebra to express the color distribution in an image with its moment. Because the color information is mainly distributed in low orders, the first-order color moment (average), the second-order color moment (variance) and the third-order color moment (skewness) are sufficient to describe the color distribution of one image. The mathematical definition is as follows (Stricker and Orengo, 1995) :

$$
\mu_{\mathrm{i}}=\frac{1}{\mathrm{~N}} \sum_{\mathrm{j}=1}^{\mathrm{N}} \mathrm{p}_{\mathrm{i}, \mathrm{j}}
$$

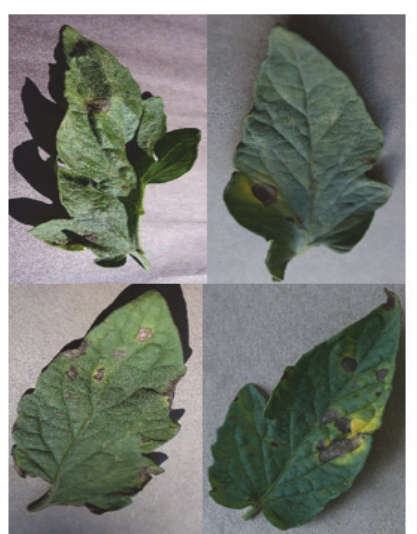

(a)

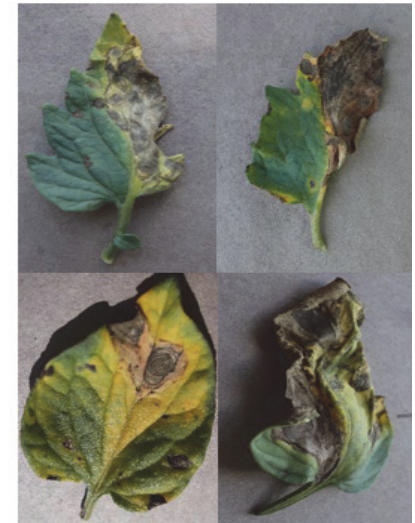

(b)
Figure 1. (a) Tomato early blight fungus general and (b) tomato early blight fungus serial. 


$$
\begin{aligned}
\sigma_{i} & =\left(\frac{1}{N} \sum_{j=1}^{N}\left(p_{i, j}-\mu_{i}\right)^{2}\right)^{\frac{1}{2}} \\
s_{i} & =\left(\frac{1}{N} \sum_{j=1}^{N}\left(p_{i, j}-\mu_{i}\right)^{3}\right)^{\frac{1}{3}}
\end{aligned}
$$

where $\mathrm{N}$ is the number of pixels $\mathrm{P}$ in the image, $\mathrm{Pij}$ are the $\mathrm{i}$ th color component of the $\mathrm{j}$-th pixel of the color image, $\mu_{\mathrm{i}}$ is the first-order color moment, $\sigma_{i}$ is the second moment of color; $\mathrm{s}_{\mathrm{i}}$ is the third moment of color. The advantage of this method is that it does not require color space quantization, and low dimension of feature vector.

The color coherence vector $(\mathrm{CCV})$ is a more complex color histogram that contains the spatial information of the color distribution to overcome disadvantage of CM model where the color histogram cannot express the spatial position of the image color. It classifies each pixel into coherence pixels or incoherence pixels. The color coherence vector (CCV) formula of image is as follows (Pass et al., 1997):

$$
<\left(a_{1}, b_{1}\right), \ldots,\left(a_{n}, b_{n}\right)>
$$

where $(a i, b i)$ is the coherence vector pair of the corresponding cluster. In this article, the aggregation threshold is 1 and the quantization level is 50 .

\section{Texture Feature Extraction}

The histogram of oriented gradients (HOG) was proposed by the French researcher DALAL in CVPR in 2005. The $\mathrm{HOG}$ is a feature descriptor in image processing for image textures. Compared with other feature description methods, the HOG feature has many advantages for disease classify. First, the HOG is operated on the local grid element of image, it maintains good invariance to the geometric and optical deformation. Second, fine directional sampling and strong local optical normalization are conducted by coarse spatial sampling, the sparse and small angular variance during acquisition process can be absorbed and classification results is improved. Its main idea is that the edge or contour of an image has a large gradient change. Therefore, the gradient direction histogram of the local area of the image is calculated to generate features. The edge or contour information of a leaf disease is described by the gradient of the pixel points $(x, y)$ in the image:

$$
\begin{aligned}
& G_{x}(x, y)=H(x+1, y)-H(x-1, y) \\
& G_{y}(x, y)=H(x, y+1)-H(x, y-1)
\end{aligned}
$$

The formulas $G_{x}(x, y), G_{y}(x, y)$, and $H(x, y)$, respectively, represent the horizontal gradient, vertical gradient and image value of pixel $(x, y)$ in the input image. The gradient amplitude and direction at pixels $(x, y)$ are calculated as:

$$
\begin{gathered}
G(x, y)=\sqrt{G_{x}(x, y)^{2}+G_{y}(x, y)^{2}} \\
\alpha(x, y)=\tan ^{-1}\left(\frac{G_{y}(x, y)}{G_{x}(x, y)}\right)
\end{gathered}
$$

In this article, the process of hog feature extraction is illustrated as follows: the sample image is divided into many cells of $64 \times 64$ pixel, and the gradient direction is divided into 9 intervals (bins) on average. In each cell, histogram statistics are calculated for the gradient direction of all pixels in each direction interval, and a 9-dimensional feature vector is obtained. Each 4 adjacent cells form a block, and the feature vectors in a block are connected to obtain 36-dimensional feature vectors. The sample image is scanned with blocks, and the scanning step is one cell. Finally, the features of all blocks are concatenated to obtain the image features.

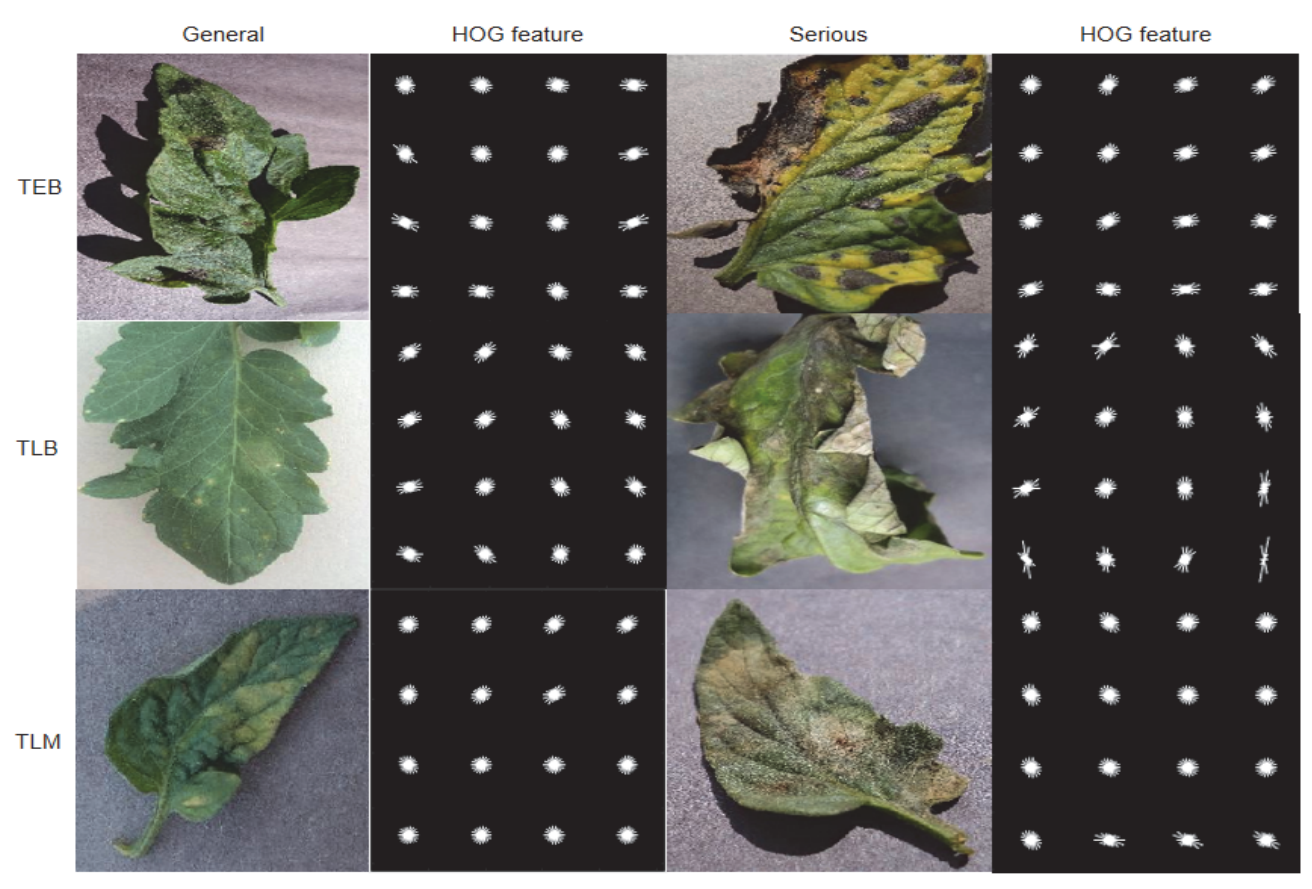

Figure 2. Textural features (HOG) of tomato leaf diseases. 
Figure 2 shows the HOG of general and serious tomato leaf diseases images in AI Challenger 2018. In figure 2, the HOG features as an array of rose plots, each rose plot shows the distribution of edge directions within a cell, the distribution is visualized by a set of directed lines whose lengths are scaled to indicate the contribution made by the gradients in that particular direction, the line directions are fixed to the bin centers of the orientation histogram and are between 0 and 360 stages measured counterclockwise from the positive $\mathrm{X}$ axis. In figure 2 , each leaf image was divided into 16 cells, the distribution of edge directions are different in 16 cells of each image. It could find that HOGs can be clearly distinguished for different edges and outlines of leaves.

\section{KMSM RECOGNITION MODEL}

The kernel mutual subspace method (KMSM) (Wolf and Shashua, 2003; Marukatat, 2016) is an appearance-based method for classifying set of images or modes. As feature patterns usually have highly nonlinear structure, the nonlinear classification problem of multiple pattern sets is required to conquer. The KMSM is a nonlinear classifier extension from the mutual subspace method (MSM) (Oja, 1983; Maeda and Watanabe, 1985) by introducing kernel equation, with potential to solve the nonlinear classification problem.

The MSM is a well-known method for object recognition based on image sets, used widely for solving various pattern recognition problems, whose input subspace was generated from a set of input patterns as a class (Watanabe, 1973). It classifies an input pattern vector into several classes, based on the minimum distance or angle between the input pattern vector in each class subspace, where a class subspace corresponds to the distribution of pattern vectors of the class in high dimensional vector space (Fukui and Yamaguchi, 2015) The relationship between two sub-spaces is strictly defined by the multiple canonical angels (Chatelin et al., 2012), which is an extension of the angle between two vectors. Therefore, in the MSM method, the structural similarity between the distributions of the image patterns is measured by the canonical angles $\theta$ between two the eigenvectors $P$ registered as a dictionary and the eigenvectors $Q$ obtained from the input data (fig. 3).

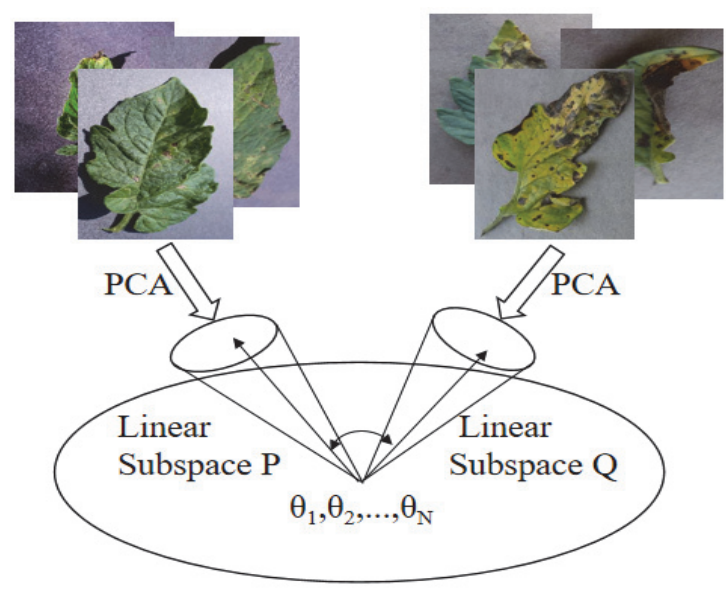

Figure 3. MSM classification model.
In the $\mathrm{m}$-dimensional feature subspace, a $\mathrm{d}_{\mathrm{p}}$-dimensional linear input subspace $\mathrm{P}$ and $\mathrm{d}_{\mathrm{q}}$-dimensional linear reference subspace $\mathrm{Q}$ are given. The definition of the canonical angles $\left\{0 \leq \theta_{1}, \ldots, \theta_{\mathrm{m}_{\mathrm{p}}} \leq \frac{\pi}{2}\right\}$ between $\mathrm{P}$ and $\mathrm{Q}$ is (Maeda and Watanabe, 1985):

$$
\cos \left(\theta_{k}\right)=\max _{u \in P} \max _{v \in Q} u^{T} v
$$

where $u_{i}^{T} u_{i}=v_{i}^{T} v_{i}=1, u_{i}^{T} u_{j}=0, v_{i}^{T} v_{j}=0, i \neq j, i=1 \sim d_{p}$, and $\mathrm{i}=\mathrm{j} \sim \mathrm{d}_{\mathrm{q}}$, and $\mathrm{d}$ is the dimensional of the subspace for recognition. $u_{i}$ and $v_{i}$ represent the $i$-th dimensional orthogonal basis vectors of sub-spaces $\mathrm{P}$ and $\mathrm{Q}$, respectively. These orthogonal basis vectors are the eigenvectors of covariance matrix $\sum_{\mathrm{i}=1}^{1} \mathrm{x}_{\mathrm{i}} \mathrm{x}_{\mathrm{i}}^{\mathrm{T}}$, which can be calculated using the 1learning pattern $\{x\}$ of each class.

In the subspace method, a subspace with d-dimensional vectors is selected according to a criterion such as the cumulative contribution rate from the eigenvectors, which are obtained by using principal component analysis (PCA) on the entered images (Fukui and Yamaguchi, 2015; Yamaguchi et al., 1998; Scholkopf et al., 1998), which is a powerful technique for extracting structure from possibly high-dimensional data sets (Schölkopf et al., 1998). It is readily performed by solving an eigenvalue problem or using iterative algorithms that estimate principal components. It is often the case that a small number of principal components is sufficient to account for most of the structure in the data, for the eigenvector with largest eigenvalue captures the most variation among training vectors, and eigenvector with smallest eigenvalue has least variation. These principal components characterize how the images data are distributed. For instance, the principal components would be the variance along the $\mathrm{x}$ - and $\mathrm{y}$-axis for image set that are Gaussian distributed in 2D space. Figures 4 and 5 show the top 20 principal component feature maps in each training datasets of tomato late blight water mold (general and serious) with

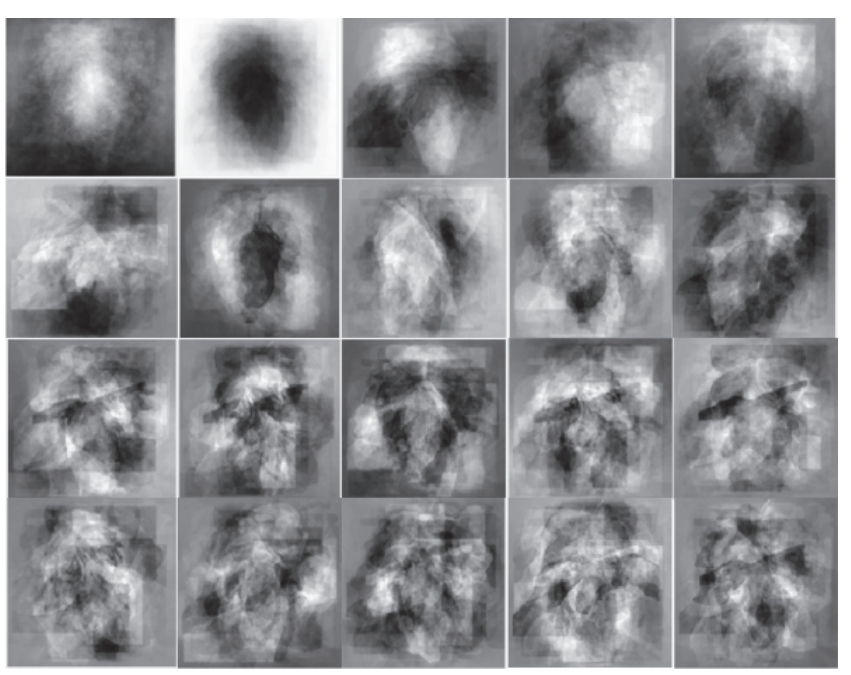

Figure 4. The first 20 principal components of TLBg in the training data. 


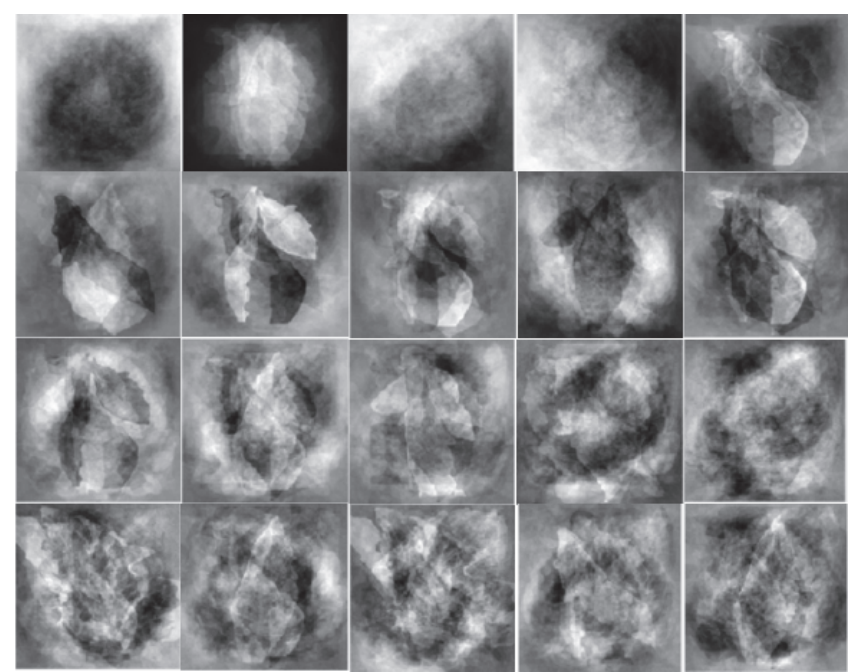

Figure 5. The first 20 principal components of TLBs in the training data.

225 training images in a 2D space. The components are ordered by their importance from top-left to bottom-right. We see that the first few components seem to primarily indicate lighting conditions, the remaining components pull out certain identifying features: shapes, textures, edge, etc.

The MSM performs well in pattern recognition, which has been utilized to compare small variations in the training data and recognition target data, and this results in a powerful recognition technique in the condition that the data distribution can be linearly approximated, when multiple data can be taken as target image inputs. To solve this problem, Wolf and Shashua (2003) proposed the kernel mutual subspace method (KMSM). In the KMSM, PCA is applied to the high-dimensional space $\mathrm{F}$ to generate a nonlinear subspace that contains the nonlinear distribution of each set of image patterns. The input pattern is mapped to a high-dimensional (in some cases infinite) feature space via nonlinear mapping. Therefore, the KMSM uses the Karenina-Loeve (KL) expansion namely PCA to perform the MSM on the nonlinear subspace generated by the mapping pattern $\{\varphi(\mathrm{x})\}$. This is a suitable method to solve the nonlinear clas-

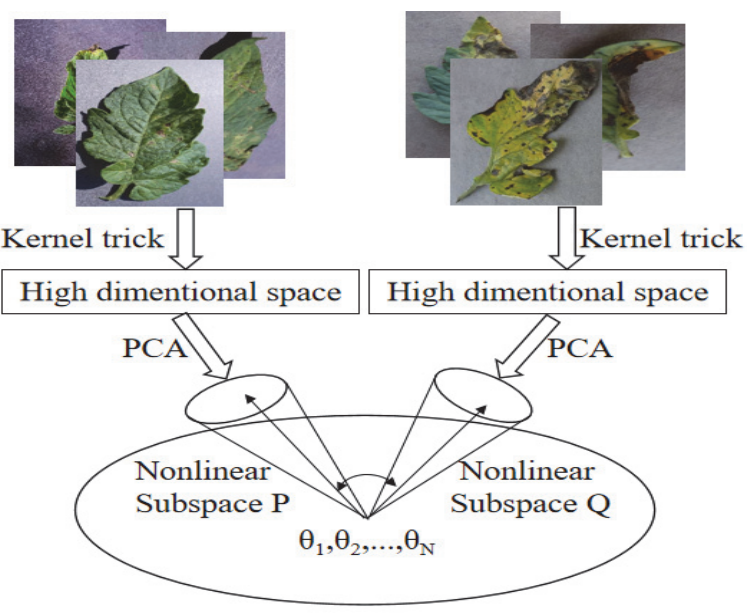

Figure 6. KMSM classification model. sification problem of multiple groups of patterns. The theoretical basis of the KMSM is kernel principal component analysis (KPCA), as shown in figure 6. KPCA is a nonlinear extension of principal component analysis that can mine the nonlinear information from datasets.

The nonlinear function $\varphi$ maps the pattern $\mathrm{x}=(\mathrm{x} 1, \ldots$, $\left.\mathrm{x}_{\mathrm{n}}\right)^{\mathrm{T}}$ of the $\mathrm{n}$-dimensional input space $\Gamma$ to the $\mathrm{f}$-dimensional feature space $\mathrm{F}: \varphi: \mathrm{R}^{\mathrm{n}} \rightarrow \mathrm{R}^{\mathrm{f}}, \mathrm{x} \rightarrow \varphi(\mathrm{x})$. To analyze the principal components of the mapped pattern, it is necessary to calculate the inner product $(\varphi(\mathrm{x}), \varphi(\mathrm{y}))$ between the function values. However, this kind of calculation is challenging because the dimension $f$ of the feature space may be very high or even infinite. If the nonlinear mapping defined by the kernel function $\mathrm{k}(\mathrm{x}, \mathrm{y})$ satisfies the Mercer condition, then the inner product $(\varphi(\mathrm{x}), \varphi(\mathrm{y}))$ can be calculated by the inner product $(\mathrm{x} \cdot \mathrm{y})$, this is called the "kernel technique."

In general, the Gaussian kernel function, which maps the samples to a higher dimensional space non-linearly, is the best choice (Sakano et al., 2010; Zhang et al., 2018). The linear kernel function is a special case of the Gaussian kernel function for linear separation. Compared with the polynomial kernel function, the Gaussian kernel function needs to determine fewer parameters. The phenotype of the sigma kernel function is very similar to that of the Gaussian kernel function with certain parameters, thus this article adopts the Gaussian kernel function, which is as follows:

$$
K_{i j}=\exp \left(-\frac{\vec{x}_{i}-\vec{x}_{j}^{2}}{\sigma^{2}}\right)
$$

where $\vec{x}_{i}$ and $\vec{x}_{j}$ are the sample image vectors, and $\sigma$ is the radius of the Gaussian kernel function.

\section{RESEARCH DESIGN FOR COLOR TEXTURE BASED KMSM METHOD}

The color texture based KMSM research approach involves two components: the learning phase and the recognition phase (fig.7). In the subspace method, multiple images were required for testing, and we noted that ten images were optimal for use in this application.

The learning phase involved three stages: First, the processed training images of each class $C \in\{1, \ldots, N\}$ were input to the system. Second, for each class, the color and texture feature matrix was calculated by extracting from each image. Finally, the KPCA was applied to the color texture feature matrix generated in the second step, establishing a nonlinear subspace as a reference subspace for each class.

The recognition phase included four stages: First, all of the collected testing images of $I_{j} \in\{1, \ldots, J\}$ were input into the system, and each I had features $\left\{\mathrm{f}_{1}, \ldots, \mathrm{f}_{\mathrm{n}}\right\}$. Second, test matrix MI was generated by extracting color and texture feature from $f_{i}$. Then, KPCA was applied to establish the nonlinear subspace for testing the subspace for each testing set. Finally, the canonical angles between the current testing subspace and each reference subspace were calculated. The current testing image set was assigned to the class with whom it shared the smallest canonical angles, which indicated that it had the high test similarity when referenced to the training datasets. 


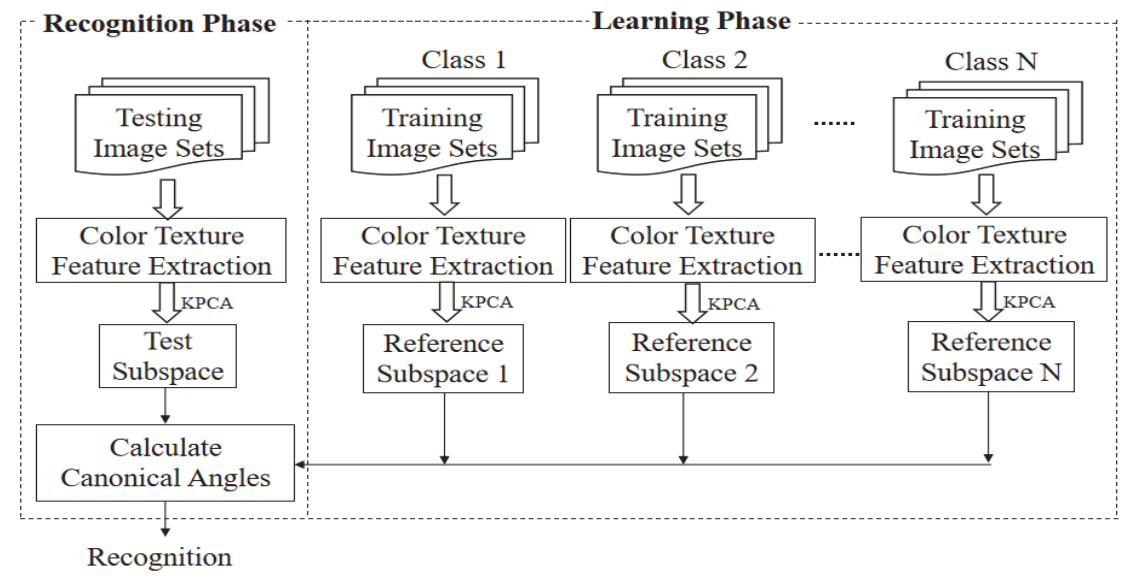

Figure 7. Flowchart of the KMSM classification model.

\section{EXPERIMENT AND RESULT ANALYSIS}

\section{EXPERIMENTAL ENVIRONMENT}

In the experiment, the tomato diseases datasets in PlantVillage datasets and AI Challenger 2018 datasets were used to validate the effectiveness of the proposed color texture KMSM model, experiments were conducted for both disease types recognition and stages of disease recognition. The evaluation was based on the computation time, the recognition rate, and the recall and F1 value. The 10-fold cross-validation was applied to evaluate the proposed model performance, the original sample is randomly partitioned into 10 equal size sub-samples $\mathrm{S}=\left\{\mathrm{S}_{\mathrm{i}}\right\}, i=1, \ldots, 10$. Of the 10 sub-samples, a single sub-sample is retained as the validation data for testing the model, and the remaining 9 subsamples as training data. The cross-validation process is then repeated 10 times, the 10 results from the folds can then be averaged to produce a single estimation.

For the setting of the experiments, the image features extracted in this article include the following: the color moment feature (CM, length:9), the color coherence vector (CCV, length:100), and the texture HOG feature (HOG, length:324). Then combined them into a $433 \times 1$ column vector $\mathrm{CM}+\mathrm{CCV}+\mathrm{HOG}$ as the color texture feature vector of each image.
In this research, the experimental platform is a $\mathrm{PC}$ with an Intel (R) core (TM) i7-7700 CPU @ 3.60 GHz and $8.0 \mathrm{~GB}$ of RAM.

\section{TOMATO Disease TyPES RECOGNITION}

The PlantVillage datasets of tomato diseases includes nine kinds of tomato diseases and one kind of healthy leaf. One thousand images were selected for each class of tomato disease datasets for a total of 10000 images (1000 images $\times$ 10 classes).

Figure 8 shows the experiment results by using the proposed method on the PlantVillage, which include three parts: the average results of the 10-fold cross-validation for each class, the best accuracy with sub-samples $\mathrm{S}_{6}$ and the average accuracy for all 10 categories AC. The results AC showed that the average recognition rate of the 10 -fold cross-validation is $98.5 \%$, and the average recognition rate of the best accuracy with sub-samples $\mathrm{S}_{6}$ is $99.34 \%$, the precision is $99.26 \%$, the recall is $99.23 \%$, the F1 score is $99.25 \%$, see as table 2. Figure 9 shows the confusion matrices of with testing sub-samples $\mathrm{S}_{6}$, it shows that a tomato bacterial spot (TBS) was recognized as tomato target spot bacteria (TTS) once, and tomato leaf mold (TLM) was mistakenly recognized as a healthy tomato $(\mathrm{TH})$ four times and tomato late blight (TLB) once.

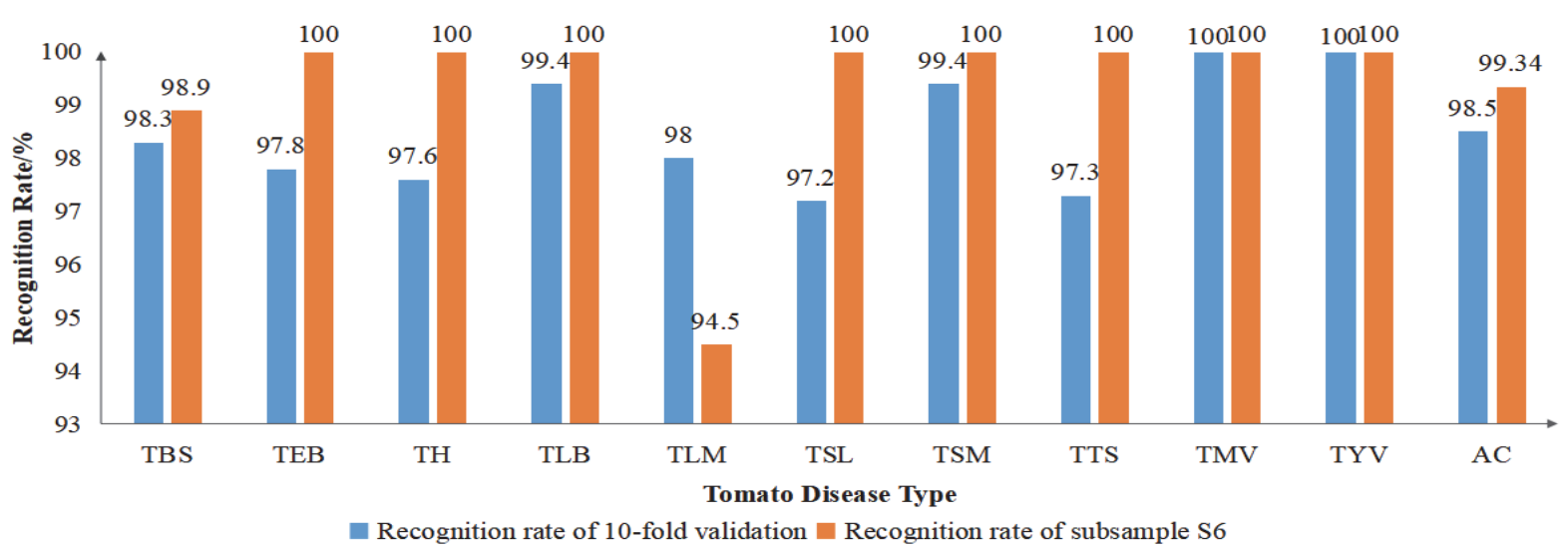

Figure 8. Comparison of the recognition rate between the validation set and sub-sample $S_{6}$ in PlantVillage. 


\begin{tabular}{|c|c|c|c|c|c|c|c|c|c|c|}
\hline & TBS & TEB & $\mathrm{TH}$ & TLB & TLMI & TSL & TSII & TTS & TUV & TYV \\
\hline TBS & $\begin{array}{c}90 \\
(98.9 \%)\end{array}$ & 0 & 0 & 0 & 0 & 0 & 0 & 1 & 0 & 0 \\
\hline TEB & 0 & $\begin{array}{c}91 \\
(100 \%)\end{array}$ & 0 & 0 & 0 & 0 & 0 & 0 & 0 & 0 \\
\hline $\mathrm{TH}$ & 0 & 0 & $\begin{array}{c}91 \\
(100 \%)\end{array}$ & 0 & 0 & 0 & 0 & 0 & 0 & 0 \\
\hline TLB & 0 & 0 & 0 & $\begin{array}{c}91 \\
(100 \%)\end{array}$ & 0 & 0 & 0 & 0 & 0 & 0 \\
\hline TLM & 0 & 0 & 4 & 1 & $\begin{array}{c}86 \\
(94.5 \%)\end{array}$ & 0 & 0 & 0 & 0 & 0 \\
\hline TSL & 0 & 0 & 0 & 0 & 0 & $\begin{array}{c}91 \\
(100 \%)\end{array}$ & 0 & 0 & 0 & 0 \\
\hline TSM & 0 & 0 & 0 & 0 & 0 & 0 & $\begin{array}{c}91 \\
(100 \%)\end{array}$ & 0 & 0 & 0 \\
\hline TTS & 0 & 0 & 0 & 0 & 0 & 0 & 0 & $\begin{array}{c}91 \\
(100 \%)\end{array}$ & 0 & 0 \\
\hline TMV & 0 & 0 & 0 & 0 & 0 & 0 & 0 & 0 & $\begin{array}{c}91 \\
(100 \%)\end{array}$ & 0 \\
\hline TYV & 0 & 0 & 0 & 0 & 0 & 0 & 0 & 0 & 0 & $\begin{array}{c}91 \\
(100 \%)\end{array}$ \\
\hline
\end{tabular}

Figure 9. Confusion matrix of the sub-sample $S_{6}$ on PlantVillage using the proposed method.

Table 2. The recognition results on sub-sample $S_{6}$ in PlantVillage using the proposed method.

\begin{tabular}{ccccc}
\hline $\begin{array}{c}\text { The Proposed } \\
\text { Method }\end{array}$ & $\begin{array}{c}\text { Accuracy } \\
(\%)\end{array}$ & $\begin{array}{c}\text { Precision } \\
(\%)\end{array}$ & $\begin{array}{c}\text { Recall } \\
(\%)\end{array}$ & $\begin{array}{c}\text { F1 Score } \\
(\%)\end{array}$ \\
\hline PlantVillenge $\mathrm{S}_{6}$ & 99.34 & 99.26 & 99.23 & 99.25 \\
\hline
\end{tabular}

\section{Stages OF TOMATo Disease ReCOgNition}

In this experiment, the AI Challenger 2018 tomato disease datasets was used, through preprocessing, 6 groups (general and serious) of tomato diseases, 1 tomato mosaic virus group and 1 healthy tomato group were selected for a total of 14 categories: TH, TEBg, TEBs, TLBg, TLBs, TLMg, TLMs, TSLg, TSLs, TSMg, TSMs, TYVg, TYVs, TMV. For the processed datasets, 250 disease pictures were selected for each category, forming a total of 3500 tomato disease images (250 images $\times 14$ classes).

Figure 10 shows the experiment results by applying the proposed method on the AI Challenger 2018. The results also include three parts: the average results of the 10-fold cross-validation for each class, the best accuracy with subsamples $S_{10}$ and the average accuracy for all 14 categories $\mathrm{AC}$. The results of $\mathrm{AC}$ show that the average recognition rate of 10 -fold cross-validation is $96.1 \%$, and the average recognition rate of the best accuracy with subsamples $S_{10}$ is
$98.66 \%$, the precision is $98.87 \%$, the recall is $91.51 \%$, the $\mathrm{F} 1$ score is $95.05 \%$ (table 3 ). In the test set sub-samples $\mathrm{S}_{10}$, only tomato late blight had a slightly lower recognition rate, and the recognition rates of the other 13 categories were $100 \%$. Figure 11 shows the confusion matrixes of the best accuracy with sub-samples $\mathrm{S}_{10}$, the results that tomato late blight general was mistaken as serious three times, compare to 224 testing images, this mistaken can be accepted, and as the mistaken happen in one disease classes, the characteristics of the disease spots were relatively similar.

\section{COMPARISON WITH DIFFERENT MODELS}

Our model is evaluated by comparing with the SVM and VGG16 networks on the PlantVillage and AI Challenger 2018 datasets, and the comparison results are shown in tables 3 and 4, which show the input pattern, model parameters, recognition rate, model training time and average testing time of each model. In this comparative experiment, there are two input patterns for the KMSM model and SVM model to verify the superiority of the color texture feature extracted in this article: pattern one is a $10 \times 10$ grayscale image, and pattern two is the color texture feature $\mathrm{CM}+$ $\mathrm{CCV}+$ HOG. The input pattern of the VGG16 model is a $224 \times 224 \times 3$ RGB image.

According to the experimental results on the PlantVillage datasets in table 4 , the recognition rate $99.34 \%$ of color texture feature + KMSM is the highest among those three models; while the recognition rate of grayscale image + KMSM $97 \%$ is the same as that of VGG16; and the recognition rate $89.2 \%$ of color texture feature + SVM is better than that of grayscale image + SVM. The results show that the three classification models have great recognition abilities for tomato disease type datasets. Among these methods, the proposed method has the best performance, and the proposed color texture feature performed well. Regarding the training time, the grayscale image + KMSM model is the quickest, which is $0.369 \mathrm{~s}$; and that of the color texture KMSM model is $0.637 \mathrm{~s}$. Both of these two models are far quicker than the SVM and VGG16. In terms of the average testing time, the color texture feature +KMSM model is $0.04 \mathrm{~s}$, which is still quicker than that of VGG16.

According to the experimental results on AI Challenger 2018 in table 5, the recognition rate of color texture feature

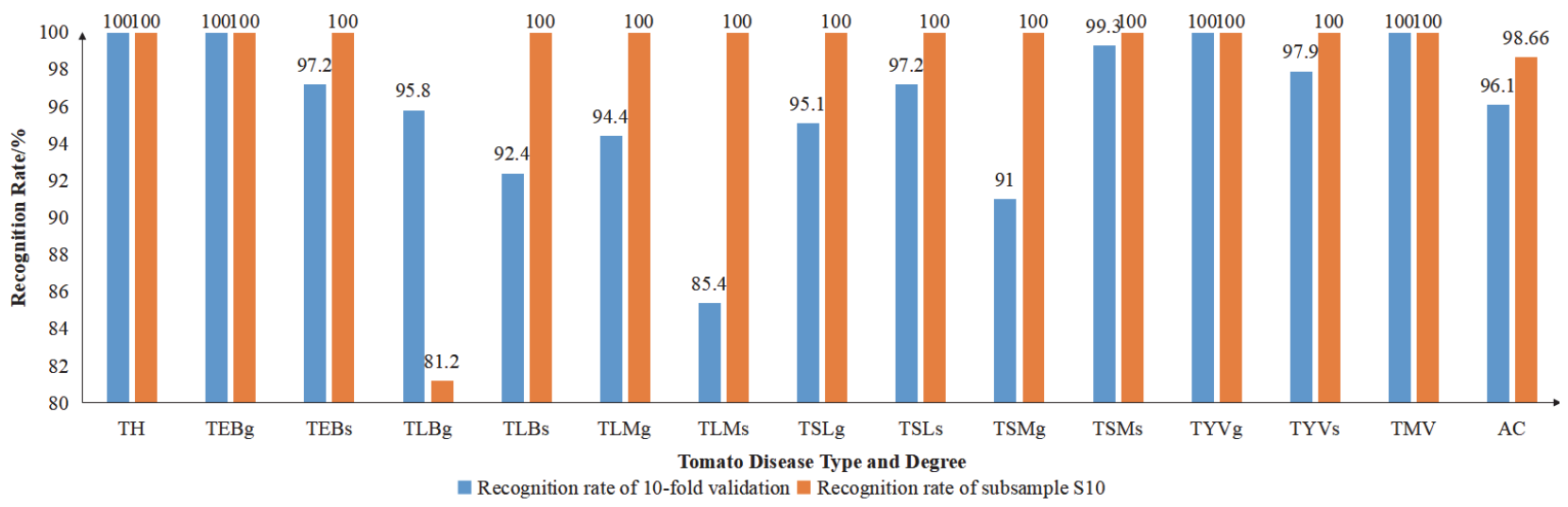

Figure 10. Comparison of the recognition rate between the validation set and sub-sample $S_{10}$ on AI Challenger 2018. 


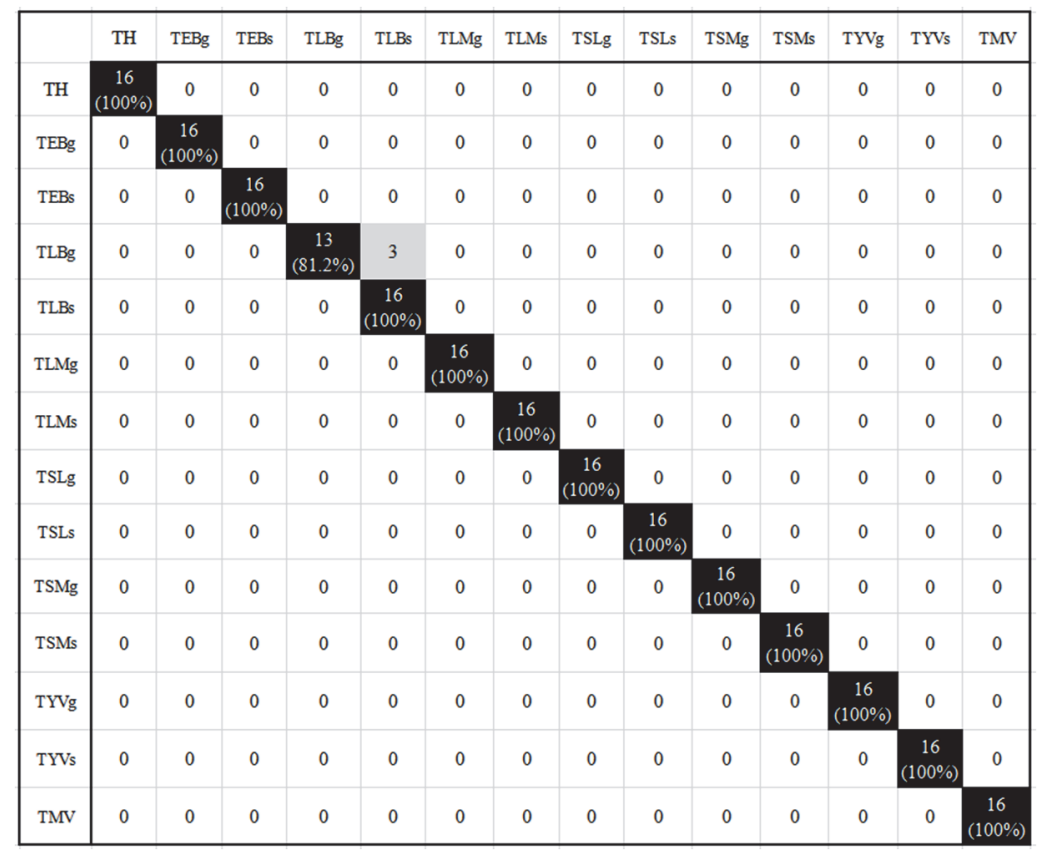

Figure 11. Confusion matrix of sub-sample $S_{10}$ on AI Challenger 2018 by using the proposed method.

Table 3. The recognition results on sub-sample $S_{10}$ in AI Challenger 2018 using the proposed method.

\begin{tabular}{ccccc}
\hline $\begin{array}{c}\text { The Proposed } \\
\text { Method }\end{array}$ & $\begin{array}{c}\text { Accuracy } \\
(\%)\end{array}$ & $\begin{array}{c}\text { Precision } \\
(\%)\end{array}$ & $\begin{array}{c}\text { Recall } \\
(\%)\end{array}$ & $\begin{array}{c}\text { F1 Score } \\
(\%)\end{array}$ \\
\hline AI $2018 \mathrm{~S}_{10}$ & 98.66 & 98.87 & 91.51 & 95.05 \\
\hline
\end{tabular}

$+\mathrm{KMSM}$ is $98.66 \%$, that of grayscale image + KMSM is $86.67 \%$, that of the SVM is $71.14 \%$ and that of VGG16 is $73.93 \%$, indicating that the SVM and VGG16 have poor recognition abilities on the stage of tomato disease data. In terms of the model training time, grayscale image + KMSM is the quickest; and the color texture feature + KMSM model takes $0.1496 \mathrm{~s}$, which is also far quicker than the SVM and VGG16. In terms of the average testing time, the grayscale image + KMSM model in this article takes $0.0047 \mathrm{~s}$, and the color texture feature + KMSM model takes $0.008 \mathrm{~s}$, which can meet the requirements of real-time system.

Compared with the SVM and VGG16, the color texture feature + KMSM model has a better recognition rate for the type and stage of tomato diseases than the SVM and VGG16; furthermore, it occupies less memory space, has a shorter training recognition time, is more stable and has higher accuracy.

\section{EXPERIMENT ON ARGUMENT IMAGES}

To verify the ability of the proposed method on diseases identification, the argument images are utilized to simulate

Table 4. Comparison of different classification models on PlantVillage.

\begin{tabular}{ccccc}
\multicolumn{5}{c}{ models on PlantVillage. } \\
\hline \multirow{3}{*}{$\begin{array}{c}\text { Recognition } \\
\text { Model }\end{array}$} & $\begin{array}{c}\text { Image } \\
\text { Feature }\end{array}$ & $\begin{array}{c}\text { Recognition } \\
\text { Rate } \\
(\%)\end{array}$ & $\begin{array}{c}\text { Training } \\
\text { Time } \\
(\mathrm{s})\end{array}$ & $\begin{array}{c}\text { Average Test } \\
\text { Time } \\
(\mathrm{s})\end{array}$ \\
\hline \multirow{2}{*}{ SVM } & Grayscale $10 \times 10$ & 69.8 & 13.956 & 0.001 \\
\cline { 2 - 5 } & $\mathrm{CM}+\mathrm{CCV}+\mathrm{HOG}$ & 89.2 & 19.663 & 0.003 \\
\hline \multirow{2}{*}{ KGG16 } & $224 \times 224 \times 3$ & 97 & 62569 & 0.185 \\
\hline \multirow{2}{*}{ KMSM } & Grayscale $10 \times 10$ & 97 & 0.369 & 0.0056 \\
\cline { 2 - 5 } & $\mathrm{CM}+\mathrm{CCV}+\mathrm{HOG}$ & 99.34 & 0.537 & 0.04 \\
\hline
\end{tabular}

Table 5. Comparison of different classification models on AI Challenger 2018.

\begin{tabular}{ccccc}
\hline $\begin{array}{c}\text { Recognition } \\
\text { Model }\end{array}$ & $\begin{array}{c}\text { Image } \\
\text { Feature }\end{array}$ & $\begin{array}{c}\text { Recognition } \\
\text { Rate } \\
(\%)\end{array}$ & $\begin{array}{c}\text { Training } \\
\text { Time } \\
(\mathrm{s})\end{array}$ & $\begin{array}{c}\text { Average Test } \\
\text { Time } \\
(\mathrm{s})\end{array}$ \\
\hline SVM & $\mathrm{CM}+\mathrm{CCV}+\mathrm{HOG}$ & 71.14 & 3.4387 & 0.002 \\
\hline VGG16 & $224 \times 224 \times 3$ & 73.93 & 39059 & 0.18 \\
\hline \multirow{2}{*}{ KMSM } & Grayscale $10 \times 10$ & 86.6 & 0.078 & 0.0047 \\
\cline { 2 - 5 } & $\mathrm{CM}+\mathrm{CCV}+\mathrm{HOG}$ & 98.66 & 0.1496 & 0.008 \\
\hline
\end{tabular}

the different camera angles and illumination condition. The experiment was verified based on AI Challenger 2018 Tomato Disease for disease stage identification. With the brightness adjustment and rotation of images, the argument images based on the $S_{1}$ was used for test-set.

The image brightness adjustment were conducted by adjust pixels value of: $+20,+40,-20$ and -40 for $R, G$, and $B$ channels, respectively. The image rotation is set with small angle and large angle, where the small angle were defined as $1^{\circ}, 2^{\circ}$, and $3^{\circ}$, and the large angle were defined as $90^{\circ}, 180^{\circ}$, and $270^{\circ}$. Through the above image argument, the $\mathrm{S}_{1}$ set was expanded to 3500 ( 25 images $\times 14$ classes $\times 10$ argument kinds), and the total number of training set images after the argument were 31500 images ( 225 images $\times 14$ classes $\times$ 10 argument kinds).

Table 6 shows the comparison of performance using $\mathrm{S}_{1}$ test-set at different rotation angles and table 7 shows the comparison of performance by increasing or decreasing of image pixels values in $\mathrm{S}_{1}$ test-set. In the table, EER (Equal Error Rate) is the Equal Error Rate, by adjusting the threshold, the False Rejection Rate (FRR) is equal to the False Acceptance Rate (FAR). As could be seen from table 6, the model proposed in this article has strong robustness to angle adjustment, and the adjusted average accuracy is $97.77 \%$, slightly lower than the original test image set. However, the average Recall and F1 values are higher than the original test 
Table 6. Comparison of experimental results for different rotation of the sample $S_{1}$.

\begin{tabular}{|c|c|c|c|c|c|c|c|}
\hline & amples & & $\begin{array}{c}\text { Accuracy } \\
(\%)\end{array}$ & $\begin{array}{c}\text { Precision } \\
(\%)\end{array}$ & EER & $\begin{array}{c}\text { Recall } \\
(\%)\end{array}$ & $\begin{array}{l}\text { F1 } \\
(\%) \\
\end{array}$ \\
\hline \multirow{6}{*}{$\begin{array}{l}\text { Rotation } \\
\text { image }\end{array}$} & \multirow{3}{*}{$\begin{array}{l}\text { Small } \\
\text { Angle }\end{array}$} & $1^{\circ}$ & 97.77 & 98.30 & 0.0455 & 97.77 & 98.03 \\
\hline & & $2^{\circ}$ & 97.77 & 98.30 & 0.0313 & 97.77 & 98.03 \\
\hline & & $3^{\circ}$ & 97.77 & 98.30 & 0.0302 & 97.77 & 98.03 \\
\hline & \multirow{3}{*}{$\begin{array}{l}\text { Large } \\
\text { Angle }\end{array}$} & $90^{\circ}$ & 97.77 & 98.30 & 0.0402 & 97.77 & 98.03 \\
\hline & & $180^{\circ}$ & 97.77 & 98.30 & 0.0362 & 97.77 & 98.03 \\
\hline & & $270^{\circ}$ & 97.77 & 98.30 & 0.0361 & 97.77 & 98.03 \\
\hline \multicolumn{3}{|c|}{ Average Value } & 97.77 & 98.3 & 0.037 & 97.77 & 98.03 \\
\hline
\end{tabular}

Table 7. Comparison of experimental results for different brightness of the sample $S_{1}$.

\begin{tabular}{|c|c|c|c|c|c|c|c|}
\hline \multicolumn{3}{|c|}{ Testing Samples } & $\begin{array}{c}\text { Accuracy } \\
(\%)\end{array}$ & $\begin{array}{c}\text { Precision } \\
(\%)\end{array}$ & EER & $\begin{array}{c}\text { Recall } \\
(\%)\end{array}$ & $\begin{array}{l}\text { F1 } \\
(\%)\end{array}$ \\
\hline \multirow{4}{*}{$\begin{array}{l}\text { Image } \\
\text { after pixel } \\
\text { value adjustment }\end{array}$} & Reduction & 20 & 95.09 & 97.09 & 0.0495 & 95.09 & 96.08 \\
\hline & value/pixels & 40 & 100 & 100 & 0.0491 & 100 & 100 \\
\hline & Increase & 20 & 95.09 & 96.83 & 0.0755 & 95.09 & 95.95 \\
\hline & value/pixel & 40 & 98.21 & 98.39 & 0.0223 & 98.21 & 98.3 \\
\hline \multicolumn{3}{|c|}{ Average Value } & 97.1 & 98.08 & 0.049 & 97.1 & 97.58 \\
\hline
\end{tabular}

data, and the ERR is lower than the original test image. In table 7 , the average accuracy is $97.1 \%$, the average precision is $98.08 \%$, the average Recall is $97.1 \%$, and the average F1 score is $97.58 \%$. Compared with the image set before argument, the recognition rate is slightly lower than that of the test image set. However, both Recall and F1 were higher than the original test image set. On the whole, the adjustment of sample pixels had little effect on the recognition of disease stage, indicating the robustness of the method proposed in this article under different illumination and different shooting positions.

\section{CONCLUSIONS AND FUTURE WORK Conclusions}

Rapid recognition of tomato disease stages is of great significance to the timely diagnosis and control of tomatoes. To recognize the stage of diseases in tomato, the Kernel Mutual Subspace Method (KMSM) is introduced in this study. The method maps the color feature and texture feature of tomato disease leaves to the high-dimensional feature space, generates a nonlinear subspace by applying principal component analysis on the generated high-dimensional feature space, and calculates the canonical angles between the generated spaces for recognition.

1. In this article, the KMSM is proposed for the fast and stable recognition of the stage of tomato diseases. Compared with the different classification models of the SVM and VGG16 on the PlantVillage and AI Challenger 2018 datasets, respectively, the experimental results show that the proposed method has the best recognition ability for the stage of tomato disease compared to those of the SVM and VGG16.

2. In the PlantVillage tomato disease type datasets, the recognition rate of color texture feature $+\mathrm{KMSM}$ is $99.34 \%$ and that of grayscale image + KMSM is $97 \%$. In the AI Challenger 2018 stage of tomato disease datasets, the recognition rate of color texture feature + KMSM is $98.66 \%$ and that of grayscale image + KMSM is $86.6 \%$, which shows that the color texture feature extracted in this article can distinguish different types and stages of diseases better.
3. In terms of the memory space and running time for the PlantVillage datasets, the training time of grayscale image + KMSM is $0.369 \mathrm{~s}$, and the average testing time is $0.0056 \mathrm{~s}$. The training time of color texture feature + KMSM is $0.537 \mathrm{~s}$. The training times of both of these methods are far lower than those of SVM (19.66 s) and VGG16 (62569 s).

4. The experimental data only include 250 images in each category from the AI Challenger 2018 tomato disease stage datasets for a total of 3500images (250 images per category $\times 14$ categories). The research results show that the proposed method can use small samples to achiever efficient and high accuracy recognition, which overcomes the problem that large samples are difficult to obtain.

5. To verify the ability of the proposed method to identify diseases using the argument images to simulate the different camera angles and illumination condition. The average accuracy slightly lower, but the average Recall and F1 values are higher than the original test data after conducted the brightness adjustment and rotation of images, indicating that the method proposed in this article has strong robustness to different camera angles and illumination condition.

The proposed method does not require a complicated model parameter training process or tedious parameter setting steps. It has the advantages of small amounts of calculations, a high disease accuracy and better stability. It can play an important role in intelligent terminal applications that have strict requirements for real-time performance, storage space and energy consumption.

\section{FUTURE WORK}

Tomato disease stages identification is of great significance for timely diagnosis and control of tomato diseases. In order to identify the disease stage of tomato quickly and stably, a rapid and stable identification method of disease stage of tomato was proposed. However, after adjustment the angle and illumination of the original image, the average accuracy is lower than that of the original image set. This is because the number of test samples is multiplied after adjust- 
ing the angle and illumination, which make the sample quality worse. Too large adjustment angle, too bright or too dark illumination condition would lead to the reduction of sample quality, so leaded to slight lower accuracy. In the future research, we will increase the diversity and number of training samples, and further improve the algorithm to improve the recognition rate.

\section{ACKNOWLEDGMENTS}

This research was funded by the Beijing Municipal Science and Technology Planning Project under Grant Z191100004019007, the National Natural Science Foundation of China grant number 61871041, the Technical System of the National Bulk Vegetable Industry, grant number CARS-23-C06

\section{REFERENCES}

Barbedo, \& Arnal, J. G. (2016). A review on the main challenges in automatic plant disease identification based on visible range images. Biosyst. Eng., 144, 52-60. https://doi.org/10.1016/j.biosystemseng.2016.01.017

Canizares, M. C., Rosas-Diaz, T., Rodriguez-Negrete, E., Hogenhout, S. A., Bedford, I. D., Bejarano, E. R., ... Moriones, E. (2015). ArabidopsisÂ thaliana, an experimental host for tomato yellow leaf curl disease-associated begomoviruses by agroinoculation and whitefly transmission. Plant Pathol., 64(2), 265-271. https://doi.org/10.1111/ppa.12270

Chatelin, F., Ahues, M., \& Ledermann, W. (2012). Eigenvalues of matrices. https://doi.org/10.1137/1.9781611972467

Dalal, N., \& Triggs, B. (2005). Histograms of oriented gradients for human detection. Proc. IEEE Computer Society Conf. on Computer Vision and Pattern Recognition, (pp. 886-893). https://doi.org/10.1109/CVPR.2005.177

Deng, X., Lan, Y., Hong, T., \& Chen, J. (2016). Citrus greening detection using visible spectrum imaging and C-SVC. Comput. Electron. Agric., 130, 177-183.

https://doi.org/10.1016/j.compag.2016.09.005

Everton, C. T., Bruno, B. M., Belete, N. A., Guimaraes, D. A., \& Piston, H. (2017). Identification of soybean foliar diseases using unmanned aerial vehicle images. IEEE Geosci. Remote. S., 14(12), 2190-2194. https://doi.org/10.1109/LGRS.2017.2743715

Fuentes, A. F., Sook, Y., Jaesu, L., \& Sun, P. D. (2018/). Highperformance deep neural network-based tomato plant diseases and pests diagnosis system with refinement filter bank. Front. Plant Sci., 9, 1162. https://doi.org/10.3389/fpls.2018.01162

Fukui, K., \& Yamaguchi, O. (1998). Facial feature point extraction method based on combination of shape extraction and pattern matching. Syst. Comput. Japan, 29(6), 49-58. https://doi.org/10.1002/(SICI)1520684X(19980615)29:6<49::AID-SCJ5>3.0.CO;2-L

Hassanien, A. E., Gaber, T., Mokhtar, U., \& Hefny, H. (2017). An improved moth flame optimization algorithm based on rough sets for tomato diseases detection. Comput. Electron. Agric., 136, 86-96. https://doi.org/10.1016/j.compag.2017.02.026

Hughes, D., \& Salathe, M. (2015). An open access repository of images on plant health to enable the development of mobile disease diagnostics. Comput. Sci.

Juan, A., Diaz-Pendon, M., \& Carmen, C. R. (2010). Tomato yellow leaf curl viruses: Menage a trois between the virus complex, the plant and the whitefly vector. Mol. Plant Pathol, 11(4), 441-450. https://doi.org/10.1111/j.13643703.2010.00618.x
Karthik, R., Hariharan, M., Anand, S., Mathikshara, P., Johnson, A., \& Menaka, R. (2020). Attention embedded residual CNN for disease detection in tomato leaves. Appl. Soft Comput., 86, 105933. https://doi.org/10.1016/j.asoc.2019.105933

Kasinathan, T., \& Uyyala, S. R. (2021). Machine learning ensemble with image processing for pest identification and classification in field crops. Neural Comput. Appl., 33(13), 7491-7504. https://doi.org/10.1007/s00521-020-05497-z

Kumar, A., \& Vani, M. (2019). Image based tomato leaf disease detection. Proc. 10th Int. Conf. on Computing, Communication and Networking Technologies, (pp. 1-6). https://doi.org/10.1109/ICCCNT45670.2019.8944692

Liu, J., \& Wang, X. (2020). Early recognition of tomato gray leaf spot disease based on MobileNetv2-YOLOv3 model. Plant Methods, 16(1), 83. https://doi.org/10.1186/s13007-020-00624-2

Luna-Benoso, B., Martinez-Perales, J. C., Cortes-Galicia, J., FloresCarapia, R., \& Silva-Garcia, V. (2021). Detection of diseases in tomato leaves by color analysis. Electronics, 10(9), 1055. https://doi.org/10.3390/electronics10091055

Mabvakure, B., Martin, D. P., Kraberger, S., Cloete, L., van Brunschot, S., Geering, A. D., ... Harkins, G. W. (2016). Ongoing geographical spread of tomato yellow leaf curl virus. Virol., 498, 257-264. https://doi.org/10.1016/j.virol.2016.08.033

Maeda, K.-i., \& Watanabe, S. (1985). A pattern matching method with local structure. Trans. IEICE, 68(3), 345-352.

Marukatat, S. (2016). Kernel matrix decomposition via empirical kernel map. Pattern Recognit. Lett., 77, 50-57. https://doi.org/10.1016/j.patrec.2016.03.031

Oja, E. (1983). Subspace methods of pattern recognition. Signal Process., 7(1), 79. https://doi.org/10.1016/0165-1684(84)900288

Pantazi, X. E., Moshou, D., \& Tamouridou, A. A. (2019). Automated leaf disease detection in different crop species through image features analysis and One Class Classifiers. Comput. Electron. Agric., 156, 96-104. https://doi.org/10.1016/j.compag.2018.11.005

Pass, G., Zabih, R., \& Miller, J. (1997). Comparing images using color coherence vectors. Proc. 4th ACM Int. Conf. on Multimedia, (pp. 65-73). https://doi.org/10.1145/244130.244148

Rangarajan, A. K., Purushothaman, R., \& Ramesh, A. (2018). Tomato crop disease classification using pre-trained deep learning algorithm. Procedia Comput. Sci., 133, 1040-1047. https://doi.org/10.1016/j.procs.2018.07.070

Sakano, H., Mukawa, N., \& Nakamura, T. (2010). Kernel mutual subspace method and its application for object recognition. Electron. Commun. Jpn., 88(6), 45-53. https://doi.org/10.1002/ecjb.20190

Scholkopf, B., Smola, A., \& Muller, K.-R. (1998). Nonlinear component analysis as a kernel eigenvalue problem. Neural Comput., 10(5), 1299-1319. https://doi.org/10.1162/089976698300017467

Singkek sci-tech. (2020). Agricultural Disease. 2018 AI Challenger [DB/OL]. (2018-12-19) [2020-3-20].

Stricker, M. A., \& Orengo, M. (1995). Similarity of color images. Proc. SPIE - The Int. Society for Optical Engineering, 2420, pp. 381-392. https://doi.org/10.1117/12.205308

Tm, P., Pranathi, A., SaiAshritha, K., Chittaragi, N. B., \& Koolagudi, S. G. (2018). Tomato leaf disease detection using convolutional neural networks. Proc. 11th Int. Conf. on Contemporary Computing, (pp. 1-5). https://doi.org/10.1109/IC3.2018.8530532

Watanabe, S. (1973). Subspace method of pattern recognition. Proc. Int. Joint Conf. on Pattern Recognition, (pp. 25-32). 
Wolf, L., \& Shashua, A. (2003). Kernel principal angles for classification machines with applications to image sequence interpretation. Proc. IEEE Computer Society Conf. on Computer Vision and Pattern Recognition. IEEE.

Yamaguchi, O., Fukui, K., \& Maeda, K. (1998). Face recognition using temporal image sequence. Proc. 3rd IEEE Int. Conf. on Automatic Face and Gesture Recognition, (pp. 318-323).

https://doi.org/10.1109/AFGR.1998.670968
Zhang, Y., Gao, P., \& Ahamed, T. (2018). Development of a rescue system for agricultural machinery operators using machine vision. Biosyst. Eng., 169, 149-164.

https://doi.org/10.1016/j.biosystemseng.2018.02.009 